\title{
Análise da postura craniocervical de crianças respiradoras bucais após tratamento postural em bola suíça
}

\author{
Craniocervical posture analysis of mouth breathing children \\ after postural treatment on swiss ball
}

Priscila Weber', Eliane Castilhos Rodrigues Corrêa², Fabiana dos Santos Ferreira ${ }^{3}$, Jovana de Moura Milanesi ${ }^{4}$, Maria Elaine Trevisan ${ }^{4,5}$

RESUMO I O estudo teve como objetivo avaliar a postura craniocervical de crianças respiradoras bucais após tratamento postural em bola suíça. Doze crianças respiradoras bucais foram submetidas a um protocolo de reeducação postural constituído por exercícios de alongamento e fortalecimento muscular sobre a bola suíça, estimulação diafragmática e alongamento dos músculos acessórios da inspiração. A postura craniocervical foi avaliada através da análise biofotogramétrica. A posição da anteriorização da cabeça foi aferida por meio do ângulo formado pelos pontos localizados no tragus direito e no processo espinhoso da sétima vértebra cervical com a linha horizontal. A curvatura cervical foi avaliada pela distância horizontal de uma linha vertical tangenciando o ápice da cifose torácica e o ponto de maior concavidade da curvatura cervical. As fotografias foram obtidas antes e após dez atendimentos. A normalidade das variáveis foi verificada a partir do teste Shapiro-Wilk. Para as comparações entre as médias foi utilizado o teste $t$ de Student para amostras dependentes admitindo-se nível de significância de 5\% (p<0,05). Verificou-se uma melhora na postura craniocervical, através da diminuição da anteriorização da cabeça $(p=0,00)$ e do restabelecimento da curvatura fisiológica da coluna cervical ( $p=0,01)$. O tratamento
ABSTRACT I The study aimed to evaluate the craniocervical posture of mouth breathing children after postural treatment on swiss ball. Twelve mouth breathing children were undergone to a postural reeducation protocol through stretching and strengthening exercises on swiss ball, diaphragmatic stimulation and stretching of the inspiratory accessory muscles. Craniocervical posture was evaluated through biophotogrammetry analysis. Forward head position was measured through an angle formed by the points in the tragus and in the spinous process of the seventh cervical vertebra with a horizontal line. Cervical column curvature was taken by the horizontal distance from a vertical line passing through the thoracic kyphosis apex to the point of the greatest cervical curvature concavity. Pictures were taken before and after ten treatment sessions. The normality of the variables was tested by Shapiro-Wilk test and the Student's $t$-test was used to determine differences in variables between assessments. It was considered a significance level of $5 \%(p<0.05)$. It was verified a significant improvement on craniocervical posture with reduction of the forward head position ( $p=0.00$ ) and restoration of cervical column physiological curvature $(p=0.01)$. The postural reeducation protocol on

\footnotetext{
Estudo desenvolvido no Laboratório de Motricidade Orofacial da Universidade Federal de Santa Maria (UFSM) - Santa Maria (RS), Brasil 'Fisioterapeuta; Mestre pelo Programa de Pós-graduação em Distúrbios da Comunicação Humana da UFSM - Santa Maria (RS), Brasil. 2Fisioterapeuta; Doutora em Biologia Bucodental pela Universidade Estadual de Campinas (UNICAMP) - Campinas (SP), Brasil; Professora do Departamento de Fisioterapia e Reabilitação e do Programa de Pós-graduação em Distúrbios da Comunicação Humana da UFSM - Santa Maria (RS), Brasil.

${ }^{3}$ Fisioterapeuta; Mestranda do Programa de Pós-graduação em Distúrbios da Comunicação Humana da UFSM - Santa Maria (RS), Brasil. ${ }^{4}$ Fisioterapeuta; Doutoranda do Programa de Pós-graduação em Distúrbios da Comunicação Humana da UFSM - Santa Maria (RS), Brasil. ${ }^{5}$ Professora do Departamento de Fisioterapia e Reabilitação da UFSM - Santa Maria (RS), Brasil.
} 
postural em bola suíça proporcionou um melhor alinhamento da postura craniocervical, provavelmente pelo equilíbrio dos músculos cervicais e maior ação diafragmática.

Descritores I fisioterapia; postura; respiração bucal; fotogrametria. swiss ball reflected on craniocervical posture alignment probably by cervical muscles equilibrium and diaphragmatic action improvement.

Keywords I physical therapy; posture; mouth breathing; photogrammetry.

\section{INTRODUÇÃO}

A postura craniocervical é mantida graças a um complexo sistema neuromuscular que trabalha constantemente para contrapor a força da gravidade e o próprio peso da cabeça que tendem a desequilibrá-la anteriormente. Assim, forças estabilizadoras provêm dos músculos cervicais posteriores, que compõem em parte a cadeia muscular posterior, e da cadeia muscular anterior, onde se encontram os músculos mastigatórios e os cervicais anteriores. Qualquer perturbação a esse sistema poderá gerar um desequilíbrio entre essas forças e consequentemente a fixação de uma posição craniocervical inadequada ${ }^{1}$.

Alguns estudos têm atribuído à obstrução nasal, juntamente ao padrão respiratório apical com consequente sobrecarga dos músculos acessórios da inspiração, a origem da perturbação ao sistema de controle postural craniocervical em crianças respiradoras bucais $(\mathrm{RB})^{2-5}$.

A obstrução do espaço aéreo nasofaríngeo, tipicamente encontrada na Síndrome do Respirador Bucal (SRB), foi associada à anteriorização da cabeça como forma de retificar o trajeto das vias aéreas e facilitar a entrada do fluxo de ar no trato respiratório inferior ${ }^{2}$. Essa alteração postural foi mais evidente em crianças $\mathrm{RB}$ de etiologia obstrutiva. Crianças $\mathrm{RB}$ de etiologia funcional e respiradoras nasais não apresentaram diferença na postura da cabeça, evidenciando a influência do fator obstrutivo na postura craniocervical.

Além disso, a resistência nasal resulta em aumento do trabalho respiratório, uma vez que ela determina a necessidade de recrutamento dos músculos acessórios da inspiração $0^{6-8}$. Consequentemente, devido à sobrecarga e ao inerente encurtamento desses músculos, particularmente do esternoclidomastóideo, o crânio é tracionado para frente e para baixo, produzindo a posição anteriorizada da cabeça. Isso justifica tanto a atividade elétrica aumentada no repouso observada nesse músculo na adoção de um padrão respiratório costal superior $^{9}$, como também explica a projeção anterior da cabeça como característica da SRB.
Considerando a complexidade biomecânica do sistema postural sob a ótica das cadeias musculares, compreende-se que a alteração no posicionamento de um único segmento poderá acarretar em acomodações compensatórias em outras partes do corpo, sempre buscando o equilíbrio do sistema ${ }^{10}$. Quanto à anteriorização da cabeça, sabe-se que ela pode estar acompanhada da retificação da curva cervical inferior, com consecutiva repercussão nas regiões dorsal e lombar ${ }^{7}$. No $R B$, isso poderá trazer consequências prejudiciais uma vez que mudanças estruturais na coluna vertebral tendem a modificar o funcionamento do músculo diafragma que, por sua vez, influenciará na deformação da caixa torácica ${ }^{8,11}$, diminuindo a capacidade respiratória e consequentemente a oxigenação ${ }^{10}$.

Considerando as implicações da alteração postural craniocervical na SRB, justifica-se a necessidade do tratamento postural com ênfase na readequação da mecânica ventilatória em crianças que respiram pela boca.

Neste contexto, a bola suíça pode ser considerada um recurso terapêutico apropriado à faixa etária, pelo seu caráter lúdico, garantindo melhor adesão de escolares ao tratamento. Ainda, por exigir um maior controle corporal, incrementa o reforço muscular promovido pelos exercícios ${ }^{3,12}$.

Assim, o objetivo deste estudo foi avaliar e comparar a postura craniocervical de crianças $\mathrm{RB}$ antes e após tratamento postural em bola suíça.

\section{MATERIAIS E MÉTODOS}

A presente pesquisa constituiu-se de um estudo experimental de caráter prospectivo, com análise quantitativa dos dados. Após a aprovação do projeto pelo Comitê de Ética em Pesquisa da Universidade Federal de Santa Maria (Protocolo no 0006.0.243.000-07), os pais e/ou responsáveis das crianças foram informados a respeito dos objetivos e procedimentos utilizados na pesquisa. Aqueles que estiveram de acordo consentiram 
na participação da criança mediante a assinatura do Termo de Consentimento Livre e Esclarecido (TCLE), conforme resolução do Conselho Nacional de Saúde (CNS) 196/96.

Participaram do grupo de estudo 12 crianças $\mathrm{RB}, 6$ meninos e 6 meninas, com média de idade de $10 \pm 1,8$ anos. Para serem incluídas na pesquisa, as crianças deveriam apresentar idade entre 8 e 12 anos e diagnóstico otorrinolaringológico de respiração bucal, obtido pelo exame composto de oroscopia, rinoscopia e otoscopia, seguidos de nasofibrofaringoscopia. Os critérios de exclusão do estudo foram: presença de comprometimento neuropsicomotor e/ou qualquer limitação física que impedisse a realização do tratamento.

\section{Avaliação da postura craniocervical}

A postura craniocervical foi avaliada por meio de registro fotográfico com análise biofotogramétrica por meio do programa $\mathrm{SAPO}^{\circledR}(0.68)$, um software de avaliação postural gratuito que permite a medida de ângulos e distâncias da postura corpora $1^{13}$. A aquisição das imagens foi feita com uma máquina fotográfica digital (Sony Cybershot 7.2 megapixels) posicionada em um tripé localizado a $2 \mathrm{~m}$ da criança e a $1 \mathrm{~m}$ de altura do chão. Previamente à análise angular e linear, as imagens fotográficas foram calibradas, por meio de recursos que o próprio software disponibiliza.

As crianças foram fotografadas em perfil direito na posição ortostática, estando com os pés paralelos, membros superiores ao longo do corpo e olhar na linha do horizonte. As referências ósseas que serviram de guias para o cálculo angular foram demarcadas com bolas de isopor, com cerca de $1 \mathrm{~cm}$ de diâmetro, envoltas por fita reflexiva e aderidas ao corpo das crianças com fita dupla face.

O alinhamento horizontal da cabeça no plano sagital, que pode detectar a posição de anteriorização, foi mensurado pelo ângulo formado pelos pontos localizados no tragus direito e no processo espinhoso da sétima vértebra cervical com a linha horizonta ${ }^{14}$. Analisando-se a projeção do ângulo é possível verificar que quanto menor o seu grau, maior é a projeção anterior da cabeça.

A curvatura da coluna cervical foi avaliada pela distância horizontal de um traço vertical tangenciando o ápice da cifose torácica, denominado plano torácico, e o ponto de maior concavidade da curvatura cervical ${ }^{15}$. Segundo Rocabado, a coluna cervical apresenta uma lordose fisiológica com valores de distância cervical entre 6 e $8 \mathrm{~cm}^{16}$ e valores inferiores demonstram uma tendência à retificação.

O ângulo, bem como a distância cervical mensurados, podem ser visualizados na Figura 1.

\section{Protocolo de tratamento postural}

As crianças foram submetidas a um protocolo de tratamento postural em bola suíça composto por exercícios de alongamento das cadeias musculares anterior e posterior e de fortalecimento dos músculos estabilizadores
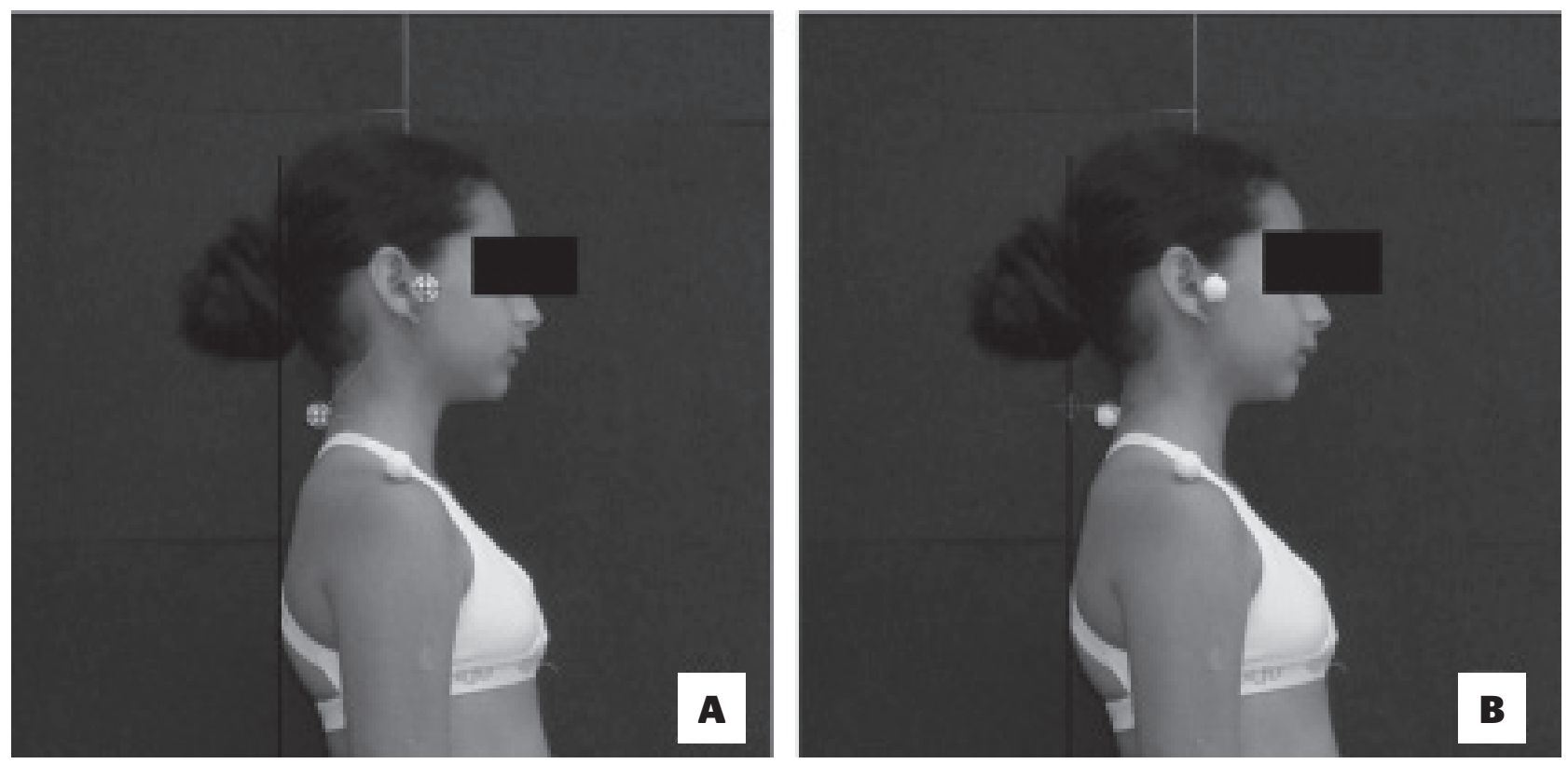

Figura 1. (A) Análise da postura craniocervical. Ângulo de anteriorização da cabeça (C7-tragus-horizontal); (B) Distância cervical 
da coluna, descrito por Corrêa e Bérzin ${ }^{12}$. Os exercícios eram realizados em vários decúbitos e consistiam em fortalecimento de músculos paravertebrais na posição prona sobre a bola (concha, extensão das costas com membros superiores fletidos ao lado da cabeça e com membros superiores abduzidos a $90^{\circ}$, extensão de membro inferior e superior de lados alternados; na posição ajoelhada com membros superiores estendidos sobre a bola (movimento da bola nos sentidos laterais direito/esquerdo e anterior/posterior). Para estabilização corporal, o programa incluiu exercícios na posição sentada sobre a bola (movimentos alternados de rotação anterior e posterior da pelve, com variações nos movimentos dos membros superiores) e exercícios abdominais. $\mathrm{Na}$ posição supina sobre a bola, eram enfatizados exercícios de alongamento (combinado a alongamento manual dos músculos escalenos, esternoclidomastóideo) e estabilização pélvica (ponte). Além disso, associou-se a reeducação do padrão respiratório por meio de estímulo manual diafragmático.

O tratamento foi realizado em 10 sessões, com frequência semanal e duração de $60 \mathrm{~min}$. As crianças foram avaliadas e reavaliadas, ao término do tratamento, pelo mesmo examinador. O tratamento foi realizado por outros fisioterapeutas que não participaram das avaliações.

\section{Análise estatística}

As análises foram realizadas por meio do software STATISTICA 7.1. A normalidade das variáveis foi verificada a partir do teste Shapiro-Wilk. Para as comparações entre as médias foi utilizado o teste $t$ de Student para amostras dependentes, admitindo-se nível de significância de $5 \%$.

\section{RESULTADOS}

Os fatores etiológicos da respiração bucal encontrados no grupo de estudo foram: rinite alérgica (62\%), hipertrofia de adenoide (30\%) e respiração bucal persistente após adenoidectomia recente (8\%).

A Tabela 1 apresenta as variáveis referentes à postura craniocervical avaliadas antes e após a décima sessão. A partir da análise dos resultados, verificou-se uma melhora da postura craniocervical com diminuição significante da anteriorização da cabeça $(p=0,00)$, medida pelo ângulo A1, e da retificação da curva cervical $(\mathrm{p}=0,01)$, medida pela distância cervical.
Tabela 1. Variáveis posturais craniocervicais avaliadas antes e após o tratamento

\begin{tabular}{|c|c|c|c|}
\hline & Pré-tratamento & Pós-tratamento & \multirow{2}{*}{ Valor $p$} \\
\hline & Média $\pm D P$ & Média $\pm D P$ & \\
\hline Ângulo A1 & $44 \pm 6,3$ & $47,8 \pm 11$ & $0,00^{*}$ \\
\hline DC & $4,4 \pm 1,05$ & $6,2 \pm 1,67$ & $0,01^{*}$ \\
\hline
\end{tabular}

DC: distância cervical em centímetros; A1: ângulo C7-tragus-horizontal em graus; DP: desvio padrão

*nivel de significância: $\mathrm{p}<0,05$

\section{DISCUSSÃO}

Neste estudo, o fator obstrutivo esteve presente na grande maioria das crianças participantes. Sabe-se que, frente à diminuição do espaço aéreo, as crianças RB tendem a readaptar o trajeto orofaríngeo para a passagem do ar, por meio de compensações posturais craniocervicais ${ }^{17}$. No entanto, considerando que as crianças estavam sob acompanhamento otorrinolaringológico para o tratamento dos seus fatores etiológicos, sugere-se que, mesmo quando tratada a causa da respiração bucal, os desequilíbrios musculares permanecem, o que pode explicar, em parte, a maior anteriorização da cabeça observada nessas crianças antes do tratamento.

A projeção anterior da cabeça presente na SRB foi observada no estudo de Lima et al. ${ }^{2}$ que comparou a postura craniocervical de crianças respiradoras bucais funcionais $(\mathrm{RBF})$ e obstrutivas $(\mathrm{RBO})$ com respiradoras nasais $(\mathrm{RN})$. Os autores concluíram que as $\mathrm{RBO}$ apresentaram uma maior anteriorização da cabeça quando comparadas às RN. Por outro lado, nenhuma diferença foi encontrada entre RBF e RN, o que reforça a influência do fator obstrutivo na postura craniocervical. Okuro et al. ${ }^{18}$, também verificaram a presença de uma anteriorização do segmento craniocervical em $96,7 \%$ de um grupo de crianças RB e 48,4\% em um grupo controle.

Por outro lado, em dois estudos recentes ${ }^{19,20}$, que também investigaram a postura cefálica por meio da análise biofotogramétrica considerando o mesmo ângulo, não foi confirmada a relação entre a respiração bucal com a presença de uma maior anteriorização da cabeça. No estudo de Bolzan et al. ${ }^{20}$, no entanto, os valores angulares para o grupo de $\mathrm{RB}$ obstrutivos $\left(45,7^{\circ}\right)$ e funcionais $\left(45,1^{\circ}\right)$ assemelharam-se aos encontrados neste estudo na avaliação prévia ao tratamento $\left(44,6^{\circ}\right)$. Em contrapartida, os autores obtiveram uma média do valor angular para o grupo de crianças $\mathrm{RN}$ de $46,1^{\circ}$, valor mais próximo ao apresentado pelas crianças deste estudo após a realização do tratamento $\left(47,8^{\circ}\right)$.

Convém salientar que não existe um valor de referência para caracterizar a posição de anteriorização da cabeça. No 
entanto, pode-se inferir, pelas medidas angulares avaliadas, que após o tratamento houve uma diminuição dessa anteriorização cefálica, argumento que também foi levado em consideração em estudos ${ }^{19,20}$ que investigaram comparativamente a postura craniocervical de indivíduos respiradores nasais e bucais.

Em relação à coluna cervical, previamente ao tratamento, as crianças apresentaram uma diminuição ou perda da lordose fisiológica, com valores inferiores (média 4,4 cm) aos de referência medidos para a distância cervical. Uma redução da lordose cervical também foi encontrada em $80 \%$ de uma amostra composta por 176 crianças RB. No entanto, os resultados foram interpretados com cautela pela autora, visto que a avaliação postural foi feita mediante análise visual, não havendo também um grupo controle para comparação ${ }^{21}$. Em outro estudo, a mesma autora procurou eliminar tais falhas metodológicas com a inclusão de um grupo de respiradores nasais e a avaliação da postura por análise biofotogramétrica. Os resultados demonstraram que as crianças $\mathrm{RB}$ apresentavam mais alterações posturais vertebrais significantes, confirmando também a retificação da curvatura cervical nesse grupo ${ }^{7}$.

Posteriormente, Cuccia et al. ${ }^{22}$ verificaram redução significante da lordose cervical em crianças $\mathrm{RB}$, quando comparadas às $\mathrm{RN}$. O referido estudo utilizou a análise cefalométrica, método mais fidedigno, sem influência dos tecidos moles na interpretação das imagens.

Após o tratamento proposto por este estudo, verificou-se uma diminuição na anteriorização da cabeça e um restabelecimento da lordose fisiológica da coluna cervical. Isso pode ser atribuído ao relaxamento e alongamento da musculatura acessória, incentivo à respiração nasal e ao estímulo diafragmático, bem como aos benefícios de realinhamento dos segmentos corporais proporcionados pelas posturas realizadas em bola suíça.

Estudos prévios de Corrêa e Bérzin ${ }^{3,12}$ demonstraram a eficácia de um tratamento de correção postural em bola suíça associado à readequação da mecânica ventilatória através do alongamento dos músculos acessórios e do estímulo diafragmático. Em um deles $^{3}$, os autores relataram que após o tratamento houve uma diminuição na atividade elétrica dos músculos esternoclidomastóideo, suboccipitais e trapézio superior, avaliados durante a inspiração nasal em crianças RB. Além disso, obtiveram uma melhora significativa na posição de anteriorização da cabeça na avaliação que sucedeu o tratamento ${ }^{12}$.

Considerando-se os resultados deste estudo, concluí-se que a intervenção fisioterapêutica seja fundamental na correção dos comprometimentos posturais envolvidos na SRB. A idade pré-puberal, que corresponde à faixa etária das crianças participantes, é considerada uma época de grandes transformações no esquema corporal que antecedem o estirão de crescimento. Essa fase também é acompanhada por uma maior elasticidade que tende a favorecer as correções das alterações posturais agravadas pela SRB e que, se não tratadas, poderão implicar em deformidades e dores musculoesqueléticas na idade adulta ${ }^{23}$.

Além disso, considerando as diversas implicações presentes na $\mathrm{SRB}$, se faz necessária uma abordagem global da criança respiradora bucal visando o controle dos fatores etiológicos pelo otorrinolaringologista, normalização das estruturas e funções estomatognáticas pelo fonoaudiólogo, correção das alterações oclusais pelo odontólogo e tratamento fisioterapêutico para uma resolução mais definitiva possível de todas as causas e consequências da respiração bucal.

Este estudo teve como limitações a falta de um grupo controle para comparação dos resultados. Além disso, considerando-se a postura corporal em seu aspecto global, se faz necessário uma abordagem das alterações que sucedem àquelas observadas na região craniocervical.

\section{CONCLUSÃO}

Os resultados do tratamento postural em bola suíça associado a estímulo manual diafragmático e alongamento dos músculos acessórios da inspiração proporcionaram melhora do alinhamento craniocervical, com diminuição da anteriorização da cabeça e do restabelecimento da lordose fisiológica da coluna cervical nas crianças $\mathrm{RB}$ participantes deste estudo.

\section{REFERÊNCIAS}

1. Armijo Olivo S, Magee DJ, Parfitt M, Major P, Norman MR. The association between the cervical spine, the stomatognathic system, and craniofacial pain: a critical review. J Orofac Pain. 2006;20(4):271-87.

2. Lima LCO, Baraúna MA, Sologurem MJJ, Canto RST, Gastaldi AC. Postural alterations in children with mouth breathing assessed by computerized biophotogrammetry. J Appl Oral Sci. 2004:12(3):232-7

3. Corrêa ECR, Bérzin F. Mouth Breathing Syndrome: Cervical muscles recruitment during nasal inspiration before and after respiratory and postural exercises on Swiss Ball. Int J Pediatr Otorhinolaryngol. 2008;72(9):1335-43. 
4. Corrêa ECR, Bérzin F. Temporomandibular disorder and dysfunctional breathing. Braz J Oral Sci. 2004:3(10):498-502.

5. Crispiniano T, Bommarito S. Avaliação da musculatura orofacial e postura corporal em pacientes com respiração bucal e maloclusão. Rev Odonto. 2007:15(29):88-97.

6. Ribeiro EC, Marchiori SC, Silva AM. Eletromiografia dos músculos esternocleidomastoideo e trapézio em crianças respiradoras bucais e nasais durante correção postural. Arq Int Otorrinolaringol. 2003;7(1):13-9.

7. Yi LC, Jardim JR, Pignatari SS, Inoue DP. The relationship between excursion of the diaphragm and curvatures of the spinal column in mouth breathing children. J Pediatr. 2008(2);84: $171-7$.

8. Hruska RJ Jr. Influences of dysfunctional respiratory mechanics on orofacial pain. Dent Clin Noth Am. 1997;41(2):211-7.

9. Cagnie B, Danneels L, Cools A, Dickx N, Cambier D. The influence of breathing type, expiration and cervical posture on the performance of the cranio-cervical flexion test in healthy subjects. Man Ther. 2008;13(3):232-8.

10. Souchard PH. Respiração. 3a ed. São Paulo: Summus Editorial; 1989.

11. Benatti AT. Equilíbrio tóraco-abdominal integrada à respiração e à postura. Arq Ciênc Saúde. 2001;5(1):87-92.

12. Corrêa ECR, Bérzin F. Efficacy of physical therapy on cervical muscle activity and on body posture in school-age mouth breathing children. Int J Pediatr Otorhinolaryngol. 2007;71(10): 1527-35.

13. Braz RG, Del Castilo Goes FP, Carvalho GA. Confiabilidade e validade de medidas angulares por meio do software para Avaliação Postural. Fisioter Mov. 2008,21(3):117-26.
14. Ferreira. EAG. Postura e controle postural: desenvolvimento e aplicação de método quantitativo de avaliação postural [tese]. São Paulo (SP): Universidade de São Paulo; 2005 [cited 2012 May 2]. Available from: http://www.teses.usp.br/teses/disponiveis/5/5160/ tde-20092006-142252/

15. Pasinato F, Corrêa ECR, Souza JA. Avaliação fotogramétrica da postura da cabeça e coluna cervical de indivíduos com disfunção temporomandibular. Ter Man. 2009;7(29):47-53.

16. Rocabado M. Biomechanical relationship of the cranio and hyoid regions. J Craniomandibular Pract. 1983;1(3):61-6.

17. Abreu RR, Rocha RL, Lamounier JA, Guerra AFM. Etiology, clinical manifestations and concurrent findings in mouth-breathing children. J Pediatr. 2008;84(6):529-35.

18. Okuro RT, Morcillo AM, Ribeiro MAGO, Sakano E, Conti PBM, Ribeiro JD. Respiração bucal e anteriorização da cabeça: efeitos na biomecânica respiratória e na capacidade de exercício em crianças. J Bras Pneumol. 2011;37(4):471-9.

19. Neiva PD, Kirkwood RN, Godinho R. Orientation and position of head posture, scapula and thoracic spine in mouth breathing children. J Pediatr Otorhinolaryngol. 2009;73(2):227-36.

20. Bolzan GP, Souza JA, Boton LM, Da Silva AMT, Corrêa ECR. Facial type and head posture of nasal and mouth breathing children. J Soc Bras Fonoaudiol. 2011:23(4):315-20.

21. Yi LC, Guedes ZCF, Pignatari S, Weckx LLM. Avaliação postural em crianças de 5 a 12 anos que apresentam respiração oral. Fisioter Mov. 2003;16(1):29-33.

22. Cuccia AM, Lotti M, Coradonna D. Oral breathing and head posture. Angle Orthod. 2008;78(1):77-82.

23. Penha PJ, Casarotto RA, Sacco ICN, Marques AP, João SMA. Qualitative postural analysis among boys and girls of seven to ten years of age. Rev Bras Fisiot. 2008;12(5):386-91. 